\title{
EXTRATO DE ALHO NA SUPERAÇÃO DA DORMÊNCIA DE PEREIRA 'HOUSUI'
}

\section{BREAK DORMANCY IN ‘HOUSUI’ PEAR USING GARLIC EXTRACT}

\author{
Odirlei Raimundo de OLIVEIRA ${ }^{1}$ \\ Bernardo LIPSKI ${ }^{2}$ \\ Éder David Borges da SILVA ${ }^{2}$ \\ Luiz Antonio BIASI ${ }^{3}$ \\ Samuel Simões COELHO²
}

\begin{abstract}
RESUMO
Com o objetivo de avaliar o efeito do extrato de alho na superação da dormência da pereira em condição de baixo acúmulo de frio foi instalado um experimento em um pomar comercial da cultivar Housui, em espaçamento 1,5 m x 4,0 m, com 7 anos de idade, no município de Campo Largo, PR. Os tratamentos foram aplicados quando as plantas estavam no estádio fenológico B (gema inchada), no dia 17/08/2007. O experimento foi realizado em delineamento inteiramente casualisado, com 3 repetições e 6 tratamentos: 1) Testemunha; 2) 4\% Óleo Mineral (OM); 3) 0,52\% Cianamida hidrogenada $(\mathrm{CH})+4 \% \mathrm{OM}$; 4) $1 \%$ Extrato de Alho (EA) + 4\% OM; 5) 2,5\% EA + 4\% OM; e 6) 5\% EA + 4\% OM; 5). As avaliações foram realizadas 29, 36, 43, 50 e 57 dias após a aplicação. Observou-se que o extrato de alho teve influencia sobre a superação da dormência das gemas da pereira. O tratamento com $5 \% \mathrm{EA}+4 \% \mathrm{OM}$ antecipou a brotação das gemas com resultado semelhante à aplicação de $0,52 \% \mathrm{CH}+4 \% \mathrm{OM}$. Nenhum tratamento apresentou efeito fitotóxico.

Palavras-chaves: Pyrus pyrifolia; cianamida hidrogenada; óleo mineral; horas de frio.
\end{abstract}

\begin{abstract}
In order to evaluate the effect of garlic extract to induce break dormancy in pear trees under low chilling conditions an experiment was installed in a 7-year-old 'Housui' pear commercial orchard located in Campo Largo, PR, presenting tree spacing with $1,5 \mathrm{~m}$ between plants $\times 4,0 \mathrm{~m}$ between rows. The treatments were applied when plants were in the $B$ (swollen bud) phenological stage which happened on 08/17/2008. A completely random experimental design of 6 treatments with 3 replications was used. Treatments were: 1) Control; 2) $4 \%$ Mineral Oil (MO); 3) $0.52 \%$ hydrogen cyanamide (HC) $+4 \% \mathrm{MO} ; 4)$ $1 \%$ Garlic Extract (GE) + 4\% MO; 5) 2.5\% GE + 4\% MO; and 6) 5\% GE + 4\% MO. The evaluations were performed 29, 36, 43, 50 and 57 days after product application. Garlic extract exerted influence on break dormancy of the pear buds. Treatment with $5 \% \mathrm{GE}+4 \% \mathrm{MO}$ anticipated bud burst, presented similar results to those obtained with $0.52 \% \mathrm{HC}+4 \%$ of $\mathrm{MO}$. No treatment was toxic to the plants.
\end{abstract}

Key-words: Pyrus pyrifolia; hydrogen cyanamide; mineral oil; chilling hours.

\footnotetext{
${ }^{1}$ Engenheiro Agrônomo autônomo, Curitiba, PR, Brasil.E-mail: odiufpr@pop.com.br

${ }^{2}$ Aluno do Curso de Agronomia, Universidade Federal do Paraná (UFPR), Curitiba, PR, Brasil. E-mail: bernardolipski@msn.com; ederdbs@gmail.com; samuelsimoescoelho@hotmail.com.

${ }^{3}$ Professor do Departamento de Fitotecnia e Fitossanitarismo. Setor de Ciências Agrárias. Universidade Federal do Paraná. Caixa Postal 19061. CEP 81531-990. Curitiba-PR, Brasil. Bolsista de Produtividade em Pesquisa do CNPq. E-mail: biasi@ufpr.br. Autor para correspondência
} 
OLIVEIRA, O.R. et al. Extrato de alho na superação da dormência...

\section{INTRODUÇÃO}

As espécies frutíferas caducifólias, quando cultivadas em regiões com acúmulo de horas de frio insuficiente para a superação da dormência, apresentam sintomas típicos de plantas mal adaptadas, como atraso e maior duração do período de floração, menor florescimento e brotação, resultando em redução na produção, com frutos desuniformes e de baixa qualidade (Marodin et al., 1992). Nessas condições, a utilização de produtos químicos, que promovam e uniformizem a brotação e a floração é prática comum na viabilização dos cultivos (Citadin et al., 2006), embora com resultados nem sempre satisfatórios (Miele et al., 1998; Nachtigal \& Camelatto, 1997). A maioria das cultivares de pereiras japonesas apresentam uma brotação e floração irregulares, demonstrando que não estão bem adaptadas às condições climáticas da região Sul do Brasil (Petri et al., 2001).

Muitos são os produtos que apresentam efeito na indução da brotação, podendo ser citados: óleo mineral, cianamida hidrogenada, dinitroortocresol, dinitrortobutilfenol, calciocianamida, thidiazuron, entre outros (Petri et al., 2002). No entanto, com a proibição do uso dos sais de dinitro, a melhor opção para a superação artificial da dormência no Brasil passou a ser a utilização de cianamida hidrogenada, associada ou não ao óleo mineral (Citadin et al., 2006). Outros compostos, como querosene, calda sulfocálcica,

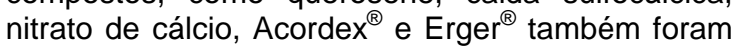
testados como indutores de brotação em macieira 'Gala' e 'Fuji', sendo que Erger ${ }^{\Theta}$ mais nitrato de cálcio apresentaram-se mais eficientes e semelhantes ao uso do tratamento padrão com óleo mineral mais cianamida hidrogenada. Entretanto, reduziram a frutificação efetiva em ambas cultivares. Já o óleo mineral mais calda sulfocálcica ou querosene mostraram-se pouco eficientes na brotação de gemas axilares (Petri, 2005).

As pesquisas realizadas demonstram que, dentre os produtos comerciais disponíveis no mercado, a cianamida hidrogenada é o mais eficiente produto químico para a superação da dormência em plantas de clima temperado (George \& Nissen, 1988; George et al., 1992; George \& Nissen, 1993; Mann et al., 1994; Mizobutsi et al., 2003). Contudo, a necessidade de restringir cada vez mais o uso de substâncias sintéticas na condução dos pomares, preconizada por sistemas sustentáveis de produção de frutas, como na Produção Orgânica e Produção Integrada (Botelho \& Müller, 2007b), torna a questão da superação da dormência de plantas frutíferas um fator limitante para a atividade no Brasil (Sanhueza et al., 2003).

No Japão, estudos sobre o efeito de compostos voláteis e não voláteis presentes em espécies do gênero Allium tem comprovado a eficiência do alho na superação da dormência de gemas de videira (Kubota et al., 2002). Kubota et al. (1999) constataram que aplicações de pasta de alho puro ou óleo de alho a $20 \%$ promoveram a brotação de gemas sem apresentar sintomas de fitotoxidez, em videiras cvs. Pione e Thompson Seedless.

No Brasil, trabalhos recentes também comprovaram a eficiência do extrato de alho na brotação de gemas de macieira e videira. Em macieira cv. Fuji Kiku, o tratamento com extrato de alho entre 1 e $10 \%+2 \%$ óleo mineral, atingiu $95 \%$ de gemas brotadas, além de antecipar a florada e a maturação dos frutos, apresentando resultados similares ao tratamento com óleo mineral mais cianamida hidrogenada (Botelho \& Müller, 2007b). O extrato de alho estimulou a brotação de $70 \%$ das gemas de ramos de videira 'Cabernet Sauvignon', que receberam mais de $168 \mathrm{~h}$ de frio abaixo de $6{ }^{\circ} \mathrm{C}$ (Botelho et al., 2007).

A ausência de resultados de pesquisa no Brasil, relativos ao efeito do extrato de alho na superação da dormência em pereira, motivou a realização deste estudo, que teve como objetivo testar o seu efeito em combinação com óleo mineral, na brotação de gemas de pereira 'Housui'.

\section{MATERIAL E MÉTODOS}

O experimento foi instalado em pomar comercial de pereira asiática (Pyrus pyrifolia), 'Housui' com 7 anos de idade, conduzido em sistema de líder central modificado, no espaçamento de $1,5 \mathrm{~m} \times 4,0 \mathrm{~m}$, localizado no município de Campo Largo, PR, latitude $25^{\circ} 25^{\prime} 24^{\prime \prime}$, longitude $49^{\circ} 32^{\prime} 37^{\prime \prime}$ e altitude $944 \mathrm{~m}$. O clima da região é classificado de acordo com KOPPEN como cfb, clima subtropical úmido mesotérmico, de verões frescos e com ocorrência de geadas severas e freqüentes, não apresentando estação seca. Durante o período de 01 de maio até 17 de agosto de 2007 , foram registradas $196 \mathrm{~h}$ de frio abaixo de $7,2 \stackrel{\circ}{\circ}$, calculadas manualmente pelo somatório das médias horárias do período, referentes a Estação Meteorológica de Pinhais, fornecidas pelo Instituto Tecnológico SIMEPAR (SIMEPAR, 2009).

O delineamento experimental utilizado foi inteiramente casualisado, com três repetições e seis tratamentos: 1) Testemunha (sem tratamento); 2) $4 \%$ Óleo Mineral (OM); 3) 0,52\% Cianamida Hidrogenada $(\mathrm{CH})+4 \% \mathrm{OM}$; 4) $1 \%$ Extrato de Alho (EA) $+4 \%$ OM; 5) 2,5\% EA + 4\% OM; e 6) $5 \% \mathrm{EA}+$ $4 \%$ OM. A unidade experimental foi constituída de uma planta, na qual foram marcados quatro ramos em posição inclinada possuindo no mínimo 12 gemas.

Os seguintes produtos comerciais foram utilizados para os tratamentos: Bioalho ${ }^{\circledR}$ (extrato de alho, Natural Rural S.A.), Triona ${ }^{\circledR}(75 \%$ óleo mineral, Bayer Cropscience S.A.) e Dormex ${ }^{\circledR}(520 \mathrm{~g}$ $\mathrm{dm}^{-3} \mathrm{H}_{2} \mathrm{CN}_{2}$, Basf S.A.). O Bioalho ${ }^{\circledR}$ é um produto natural obtido da extração por prensagem a frio de alho, sendo totalmente solúvel em água.

Os tratamentos foram aplicados quando as gemas encontravam-se no estádio fenológico B (gema inchada) (Faoro, 2001a), dia 17/08/2007, com o uso de um pulverizador costal utilizando um volume de calda de $500 \mathrm{dm}^{3}$ planta $^{-1}$. 
OLIVEIRA, O.R. et al. Extrato de alho na superação da dormência...

As avaliações ocorreram semanalmente no período de 15/09/07 à 13/10/07. Durante as avaliações foi contabilizado em cada ramo, o número total de gemas laterais, gemas com inicio de brotação (caracterizadas pela abertura das escamas e início do aparecimento de folhas ou flores), gemas brotadas (com folhas inteiras visíveis) e gemas que emitiram cachos florais e/ou frutos.

Os dados obtidos foram submetidos à análise de variância $(P=0,05)$ e quando verificada significância, procedeu-se à comparação de médias pelo teste de Tukey $(P=0,05)$.

\section{RESULTADOS E DISCUSSÃO}

O extrato de alho apresentou efeito fisiológico como indutor de brotação em gemas de pereira. $\mathrm{Na}$ avaliação das gemas no estádio de início da brotação, foi observado que a aplicação de
$5 \% \mathrm{EA}+4 \% \mathrm{OM}$ estimulou uma alta porcentagem de gemas (59,3\%), já aos 29 dias após a aplicação, sendo superior aos demais tratamentos, mas não diferindo do tratamento com $0,52 \% \mathrm{CH}+4 \% \mathrm{OM}$ (Tabela 1). Botelho \& Müller (2007b) estudando o efeito do EA em gemas de macieiras 'Fuji Kiku', também obtiveram maior brotação aos 30 dias após a aplicação, com $5 \% \mathrm{EA}+2 \% \mathrm{OM}$, seguido por uso de $10 \% \mathrm{EA}+2 \% \mathrm{OM}$ e $0,4 \% \mathrm{CH}+4 \% \mathrm{OM}$. Em outro experimento, na superação da dormência de macieira 'Royal Gala' a aplicação de 1 ou $5 \%$ EA + $2 \% \mathrm{OM}$ apresentou efeito similar ao tratamento com $0,4 \% \mathrm{CH}+4 \%$ OM. Já o tratamento com $10 \% \mathrm{EA}+$ $2 \%$ OM foi superior aos demais, com 95\% de gemas brotadas após 50 dias da aplicação. Este tratamento também foi mais efetivo na antecipação do florescimento, mas que infelizmente coincidiu com um período de excesso de chuva no início da primavera, prejudicando a produtividade de maçãs (Botelho \& Muller, 2007a).

TABELA 1 - Porcentagem de gemas em início de brotação de pereira 'Housui' após tratamento com óleo mineral (OM), extrato de alho (EA) e cianamida hidrogenada (CH). Campo Largo, PR. 2007.

\begin{tabular}{|c|c|c|c|c|c|}
\hline \multirow{3}{*}{ Tratamento } & \multicolumn{5}{|c|}{ Dias após a pulverização } \\
\hline & 29 & 36 & 43 & 50 & 57 \\
\hline & $15 / 9 / 2007$ & $22 / 9 / 2007$ & $29 / 9 / 2007$ & $6 / 10 / 2007$ & $13 / 10 / 2007$ \\
\hline & \multicolumn{5}{|c|}{ Gemas em início de brotação (\%) } \\
\hline Testemunha & $27,8 b^{*}$ & $25,0 a b$ & 6,5 a & $3,4 \quad a$ & 0,7 a \\
\hline $4 \% \mathrm{OM}$ & $31,6 \mathrm{~b}$ & 32,8 a & 7,4 a & $4,3 \quad a$ & 0,6 a \\
\hline $0,52 \% \mathrm{CH}+4 \% \mathrm{OM}$ & $43,1 a b$ & $6,6 \mathrm{~b}$ & 8,4 a & 0,0 a & 0,0 a \\
\hline $1,0 \% \mathrm{EA}+4 \% \mathrm{OM}$ & $31,0 \mathrm{~b}$ & $16,6 \mathrm{ab}$ & 2,7 a & 2,5 a & 0,8 a \\
\hline $2,5 \% \mathrm{EA}+4 \% \mathrm{OM}$ & $37,9 \mathrm{~b}$ & $25,7 a b$ & 6,9 a & $1,7 \mathrm{a}$ & 0,0 a \\
\hline $5,0 \% \mathrm{EA}+4 \% \mathrm{OM}$ & 59,3 a & $16,3 \mathrm{ab}$ & 3,5 a & $1,8 \mathrm{a}$ & 0,0 a \\
\hline Média & 38,4 & 20,5 & 5,9 & 2,3 & 0,3 \\
\hline CV (\%) & 18,3 & 22,7 & 36,2 & 42,4 & 28,2 \\
\hline
\end{tabular}

Dados transformados em raiz quadrada de $x+1$ para análise.

${ }^{\star}$ Médias seguidas por letras iguais não diferem entre si pelo teste Tukey $(P=0,05)$.

O efeito fisiológico do extrato de alho na indução da brotação de gemas é atribuído à presença de compostos voláteis a base de enxofre, unidos com grupos alil $\left(\mathrm{CH}_{2} \mathrm{CHCH}_{2}\right)$, com destaque ao dialil dissulfito, que é o sulfito encontrado no alho (Kubota et al., 1999).

O tratamento com 5\% EA + 4\% OM antecipou o início da brotação das gemas. Nas avaliações posteriores a porcentagem de gemas nesse estádio caiu abruptamente, consequência da evolução da maior parte delas para o estádio de gema brotada. A aplicação de $0,52 \% \mathrm{CH}+4 \% \mathrm{OM}$ apresentou a mesma tendência, sendo que na primeira avaliação, esse tratamento já apresentou $24,79 \%$ de gemas brotadas, contra $16,39 \%$ do tratamento com 5\% EA + 4\% OM (Tabela 2), confirmando a eficiência da cianamida hidrogenada na superação da dormência de gemas e antecipação da brotação. Aos 50 dias após a aplicação, apenas nesse tratamento não foram encontradas mais gemas em inicio de brotação, demonstrando que a $\mathrm{CH}$ foi mais eficiente na uniformização da brotação. A concentração da brotação é desejável, sendo um dos objetivos da quebra da dormência, pois a uniformidade das fases fenológicas facilita e aumenta a eficiência do manejo do pomar, tornando a fruticultura racional e rentável.

Até 36 dias após a aplicação dos produtos, a porcentagem de gemas brotadas foi superior nos tratamentos com 2,5 e $5 \%$ EA + $4 \%$ OM e $0,52 \%$ $\mathrm{CH}+4 \%$ OM. Nas avaliações posteriores, a porcentagem de brotação dos tratamentos com apenas 4\% OM e testemunha, cujas gemas apresentaram brotação mais lenta, se elevaram não apresentando mais diferença significativa com os demais, com exceção ao tratamento $1 \% E A+4 \%$ $\mathrm{OM}$, que sempre apresentou os menores valores de brotação (Tabela 2). 
OLIVEIRA, O.R. et al. Extrato de alho na superação da dormência...

TABELA 2 - Porcentagem de gemas brotadas de pereira 'Housui' após tratamento com óleo mineral (OM), extrato de alho (EA) e cianamida hidrogenada (CH). Campo Largo, PR. 2007.

\begin{tabular}{|c|c|c|c|c|c|}
\hline \multirow{3}{*}{ Tratamento } & \multicolumn{5}{|c|}{ Dias após a pulverização } \\
\hline & 29 & 36 & 43 & 50 & 57 \\
\hline & $15 / 9 / 2007$ & $22 / 9 / 2007$ & $29 / 9 / 2007$ & $6 / 10 / 2007$ & $13 / 10 / 2007$ \\
\hline & \multicolumn{5}{|c|}{ Gemas brotadas (\%) } \\
\hline Testemunha & $1,52 c^{*}$ & $24,34 \quad b$ & $48,70 a b$ & $53,73 a b$ & $57,60 \mathrm{ab}$ \\
\hline $4 \% \mathrm{OM}$ & 4,08 bc & 26,32 b & $57,78 a b$ & $60,40 a b$ & 65,54 a \\
\hline $0,52 \% \mathrm{CH}+4 \% \mathrm{OM}$ & 24,79 a & $41,62 a b$ & $55,33 a b$ & 64,88 a & 66,41 a \\
\hline $1,0 \% \mathrm{EA}+4 \% \mathrm{OM}$ & $1,39 \mathrm{c}$ & $24,64 b$ & $41,29 b$ & $42,27 \quad b$ & $43,84 \quad b$ \\
\hline $2,5 \% \mathrm{EA}+4 \% \mathrm{OM}$ & $5,97 \quad a b c$ & $34,43 a b$ & $61,27 a b$ & 67,75 a & 69,56 a \\
\hline $5,0 \% \mathrm{EA}+4 \% \mathrm{OM}$ & $16,39 a b$ & 55,74 a & 70,42 a & 72,25 a & $77,97 \quad$ a \\
\hline Média & 9,02 & 34,52 & 55,80 & 60,21 & 63,49 \\
\hline CV (\%) & 146,65 & 38,17 & 20,73 & 20,20 & 19,99 \\
\hline
\end{tabular}

Dados transformados em raiz quadrada de $x+1$ para análise.

${ }^{*}$ Médias seguidas por letras iguais não diferem entre si pelo teste Tukey $(P=0,05)$.

Aos 43 dias houve uma estabilização da brotação das gemas, com pequenos aumentos nas avaliações feitas aos 50 e 57 dias. Na última avaliação, observou-se cerca de $78 \%$ de gemas brotadas no tratamento com 5\% EA + 4\% OM, enquanto na testemunha havia $57,6 \%$. Esperava-se uma menor brotação nas plantas que não receberam os produtos, pois a exigência em frio da cultivar Housui é de 721 h (Faoro, 2001b), mas o frio acumulado foi suficiente para promover a superação da dormência no nível observado. Também o fato de terem sido escolhidos ramos em posição inclinada, deve ter favorecido a brotação pelo menor efeito da paradormência induzida pela brotação antecipada das gemas apicais. Num experimento com pereiras da mesma cultivar, realizado em Pinhais (PR), foi observado nas plantas da testemunha, uma porcentagem de brotação de $48 \%$ após 84 dias da aplicação dos tratamentos, semelhante à observada neste trabalho, enquanto o tratamento com $4 \%$ de OM foi superior à testemunha e estimulou a brotação de $72 \%$ e $80 \%$ de gemas nos sistemas de condução em taça e líder central modificado, respectivamente (Oliveira et al., 2008). A superioridade do óleo mineral encontrada naquele experimento, provavelmente ocorreu devido ao pouco frio acumulado $(141 \mathrm{~h})$ durante o período de repouso em 2005, ano em que foi realizado o experimento. Para a pereira 'Packham's Triumph', a aplicação de $4 \%$ OM promoveu a brotação de $41,3 \%$ de gemas laterais, enquanto na testemunha ocorreu apenas $8,34 \%$ de brotação no ano de 1991, quando o somatório de horas de frio foi de $401 \mathrm{~h}$ em Eldorado do Sul-RS (Francisconi et al., 1992). No ano anterior, com a mesma cultivar no mesmo local, com maior ocorrência de frio (647 h), a brotação foi de $55,14 \%$ na testemunha e $56,8 \%$ com o tratamento com 4\% OM (Marodin et al., 1992).

Com relação à porcentagem de gemas que emitiram cachos florais e/ou frutos não foi evidenciado diferenças significativas entre os tratamentos em nenhuma data de avaliação (Tabela 3). Como a diferenciação floral das gemas ocorre no ciclo anterior, os tratamentos não tem efeito direto sobre a formação de novas flores, mas apenas sobre a abertura das gemas. A maior brotação de gemas laterais deverá permitir a maior formação de ramos frutíferos, aumentado o florescimento nos anos seguintes à aplicação dos tratamentos de superação da dormência (Francisconi et al., 1992). Houve diminuição da porcentagem de gemas que emitiram cachos florais e/ou frutos a partir da segunda avaliação, que pode ser considerado normal para a pereira, pois muitas flores que se abrem não são polinizadas ou abortam, reduzindo a frutificação efetiva (Faoro, 2001a). Na última avaliação a redução foi maior devido ao raleio de frutos realizado pelo produtor antes da avaliação, por isso não foi realizada análise estatística nessa data.

\section{CONCLUSÕES}

A aplicação de $5 \%$ de extrato de alho acrescido de $4 \%$ de óleo mineral antecipou a brotação das gemas e teve efeito similar a aplicação de $0,52 \%$ de cianamida hidrogenada com $4 \%$ de óleo mineral.

\section{AGRADECIMENTOS}

Os autores agradecem ao Eng. Agrônomo Paulo Carlos Cosmo pela concessão do pomar e de produtos para a realização deste estudo. 
OLIVEIRA, O.R. et al. Extrato de alho na superação da dormência...

TABELA 3 - Porcentagem de gemas que emitiram cachos florais e/ou frutos de pereira 'Housui' após tratamento com óleo mineral $(\mathrm{OM})$, extrato de alho $(\mathrm{EA})$ e cianamida hidrogenada $(\mathrm{CH})$. Campo Largo, PR. 2007.

\begin{tabular}{|c|c|c|c|c|c|}
\hline \multirow{3}{*}{ Tratamento } & \multicolumn{5}{|c|}{ Dias após a pulverização } \\
\hline & 29 & 36 & 43 & 50 & 57 \\
\hline & $15 / 9 / 2007$ & $22 / 9 / 2007$ & 29/9/2007 & $6 / 10 / 2007$ & $13 / 10 / 2007$ \\
\hline & \multicolumn{5}{|c|}{ Cachos florais e/ou frutos (\%) } \\
\hline Testemunha & 7,05 a & $22,81 \quad a$ & 22,89 a & 21,36 a & 18,52 \\
\hline $4 \% \mathrm{OM}$ & 11,07 a & 28,29 a & 26,58 a & 21,30 a & 7,31 \\
\hline $0,52 \% \mathrm{CH}+4 \% \mathrm{OM}$ & 12,79 a & 25,46 a & 21,90 a & 13,85 a & 9,85 \\
\hline $1,0 \% \mathrm{EA}+4 \% \mathrm{OM}$ & 17,42 a & 19,53 a & 17,67 a & 16,45 a & 9,54 \\
\hline $2,5 \% \mathrm{EA}+4 \% \mathrm{OM}$ & 9,48 a & $13,57 \mathrm{a}$ & 12,18 a & 9,58 a & 4,85 \\
\hline $5,0 \% \mathrm{EA}+4 \% \mathrm{OM}$ & $10,13 \mathrm{a}$ & 14,05 a & 14,36 a & 11,16 a & 3,89 \\
\hline Média & 11,32 & 20,62 & 19,26 & 15,62 & 8,99 \\
\hline $\mathrm{CV}(\%)$ & 89,39 & 62,21 & 58,57 & 69,36 & - \\
\hline
\end{tabular}

Dados transformados em raiz quadrada de $x+1$ para análise.

*Médias seguidas por letras iguais não diferem entre si pelo teste Tukey $(P=0,05)$.

\section{REFERÊNCIAS}

1. BOTELHO, R. V.; MÜLLER, M. M. L. Evaluation of garlic extract on bud dormancy release of 'Royal Gala' apple trees. Australian Journal of Experimental Agriculture, v. 47, n. 6, p. 738-741, 2007 a.

2. BOTELHO, R. V.; MÜLLER, M. M. L. Extrato de alho como alternativa na quebra de dormência de gemas em macieiras cv. Fuji Kiku. Revista Brasileira de Fruticultura, v. 29, n. 1, p. 37-41, 2007b.

3. BOTELHO, R. V. et al. Effects of chilling and garlic extract on bud dormancy release in Cabernet Sauvignon grapevine cuttings. American Journal Enology and Viticulture, v. 58, n. 3, p. 402-404, 2007.

4. CITADIN, I. et al. Uso de cianamida hidrogenada e óleo mineral na floração, brotação e produção do pessegueiro 'Chiripá'. Revista Brasileira Fruticultura, v. 28, n. 1, p. 32-35, 2006.

5. FAORO, I. D. Morfologia e fisiologia. In: EPAGRI. Nashi, a pêra japonesa. Florianópolis: Epagri/Jica, 2001a, p. 67-94.

6. FAORO, I. D. Cultivares e porta-enxertos. In: EPAGRI. Nashi, a pêra japonesa. Florianópolis: Epagri/Jica, 2001b, p. 95138.

7. FRANCISCONI, A. H. D. et al. Efeito de óleo mineral, cianamida hidrogenada e thidiazuron na quebra de dormência e produção da pereira (Pyrus communis L.) Cv. Packham's Triumph. Revista Brasileira de Fruticultura, v. 14, n. 1, p. 161166, 1992.

8. GEORGE, A. P.; LLOYD, J.; NISSEN, R.J. Effects of hydrogen cyanamide, paclobutrazol and pruning date on dormancy release of the chill peach cultivar Flordaprince in subtropical Australia. Australian Journal of Experimental Agriculture, v. 32, n. 1, p. 89-95, 1992.

9. GEORGE, A. P.; NISSEN, R. J. Chemical methods on breaking dormancy of low chill nectarines: preliminary evaluations in subtropical Queensland. Australian Journal of Experimental Agriculture, v. 28, n. 3, p. 425-429, 1988.

10. GEORGE, A. P.; NISSEN, R. J. Effects of growth regulants on defoliation, flowering, and fruit maturity of the low chill peach cultivar Flordaprince in subtropical Australia. Australian Journal of Experimental Agriculture, v. 33, n. 6, p. 787795, 1993.

11. KUBOTA, N.; YAMANE, Y.; TORIU, K. Breaking dormancy in grape cuttings with non-volatile and volatile compounds of several Allium species. Journal of the Japanese Society for Horticultural Science, v. 71, n. 4, p. 467-472, 2002.

12. KUBOTA, N. et al. Identification of active substances in garlic responsible for breaking bud dormancy in grapevines. Journal of the Japanese Society for Horticultural Science, v. 68, n. 6, p. 1111-1117, 1999.

13. MANN, S. et al. Effect of cyanamide on bud burst, flowering and fruit maturity of Baggugosha pear. Acta Horticulturae, v. 367, p. 214-223, 1994.

14. MARODIN, G. A. B.; FRANCISCONI, A. H. D.; GALLOIS, E. S. P. Efeito de produtos químicos na quebra de dormência e produção de pereira (Pyrus communis, L.) cv. Packham's Triumph. Revista Brasileira de Fruticultura, v. 14, n. 1, p. 155$160,1992$.

15. MIELE, A.; RIZZON, L. A.; DALL'AGNOL, I. Efeito da época e do número de aplicações de cianamida hidrogenada e óleo mineral na quebra de dormência da videira. Revista Brasileira de Fruticultura, v. 20, n. 2, p. 183-187, 1998.

16. MIZOBUTSI, G. P. et al. Efeito da aplicação de cianamida hidrogenada e de óleo mineral em caquizeiro. Revista Brasileira de Fruticultura, v. 25, n. 1, p. 89-92, 2003.

17. NACHTIGALL, G. R.; CAMELATTO, D. Efeito da mistura de nitrato de cálcio com thidiazuron, cianamida hidrogenada e óleo mineral na superação da dormência de macieiras (Malus domestica, Borkh.) cv. Gala. Revista Brasileira de Fruticultura, v. 19, n. 3, p. 275-282, 1997.

18. OLIVEIRA, O.R. de et al. Quebra de dormência de pereira 'Hosui' com uso de óleo mineral em dois tipos de condução. Revista Brasileira de Fruticultura, v. 30, n.2, p. 409-413, 2008.

19. PETRI, J. L. Alternativas Para Quebra de Dormência em Fruteiras de Clima Temperado. In: ENCONTRO NACIONAL SOBRE FRUTICULTURA DE CLIMA TEMPERADO, 9., 2005. Fraiburgo. Anais... Caçador: Epagri, v. 1 (Palestras), 2005. p. 303-311. 
OLIVEIRA, O.R. et al. Extrato de alho na superação da dormência...

20. PETRI, J. L.; LEITE, G. B.; FAORO, I. D. Tratos culturais. In: EPAGRI. Nashi, a pêra japonesa. Florianópolis: Epagri/ Jica, 2001. p. 195-210.

21. PETRI, J. L.; PALLADINI, L.. A.; POLA, A. C. Dormência e indução da brotação da macieira. In: EPAGRI. Manual da cultura da macieira. Florianópolis, 2002. p. 261-298.

22. SANHUEZA, R. M. V.; ANDRIGUETO, J. R.; KOSOSKI, A. R. Situação atual da produção integrada de frutas no Brasil. In: MELO, G.W.B.; SEBBEN, S.S. (Eds.). SEMINÁRIO BRASILEIRO DE PRODUÇÃO INTEGRADA DE FRUTAS, 5., 2003, Bento Gonçalves. Anais... Bento Goncalves: Embrapa-CNPUV, 2003. p. 23-25.

23. SIMEPAR - Instituto Tecnológico SIMEPAR. Temperatura horária: Curitiba, Fevereiro de 2009. Arquivo eletrônico. Word for Windows. 2009.

Recebido em 20/01/2009 Aceito em 13/05/2009 